\title{
Framework CAP 1.0 para criação e uso de arquiteturas pedagógicas
}

\author{
Luiz F. Reinoso ${ }^{1}$, Marcello Amorim ${ }^{3}$, Orivaldo L. Tavares ${ }^{3}$, Renan F. Almeida ${ }^{2}$ \\ ${ }^{1}$ Coordenadoria de informática - Instituto Federal de Educação, Ciência e Tecnologia \\ do Espírito Santo (IFES) \\ Colatina - ES - Brasil \\ ${ }^{2}$ Coordenadoria de informática - Instituto Federal de Educação, Ciência e Tecnologia \\ do Espírito Santo (IFES) \\ Vitória - ES - Brasil \\ ${ }^{3}$ Departamento de Informática - Universidade Federal do Espírito Santo (UFES) \\ Vitória - ES - Brasil \\ luizfreinoso@gmail.com,novaes@inf.ufes.br, tavares@inf.ufes.br, ralmeida@ifes.edu.br
}

\begin{abstract}
This paper presents the framework CAP 1.0 (Constructor for Pedagogical Architectures), as well as the CAP model. This CAP 1.0 is a framework that allows you to instantiate different constructors of pedagogical architectures (CAP). CAPs can be used to generate pedagogical architectures (PA) with different characteristics and applications. To demonstrate the instantiation process of the CAPs and their subsequent use for the construction of PAs, two constructors of pedagogical architecture will be presented: CAP-MVLIBRAS, a Constructor of Pedagogical Architectures to Learn LIBRAS (Brazilian Sign Language); and CAPCOM, a constructor of pedagogical architectures for learning operations over multimedia data.
\end{abstract}

Resumo. Este artigo apresenta o framework CAP 1.0 (Gerador de CAPS), assim como o modelo CAP (Construtor de Arquiteturas Pedagógicas). O CAP 1.0 é um framework que permite instanciar diferentes CAPs, que podem ser utilizados para criar arquiteturas pedagógicas (AP) com diferentes características e aplicações. Para demonstrar o processo de instanciação dos CAPs, e seu posterior uso na construção de APs, são apresentados dois construtores de arquitetura pedagógica: o CAP-MVLIBRAS, um Construtor de Arquiteturas Pedagógicas para a Aprendizagem de Libras (Lingua Brasileira de Sinais); e o CAPCOM, um Construtor de Arquiteturas Pedagógicas para a Aprendizagem de Operações sobre Dados Multimídia.

\section{Introdução}

As arquiteturas pedagógicas (AP) são estruturas elaboradas a partir de conceitos construtivistas, e que utilizam recursos digitais de forma a facilitar a aprendizagem (Carvalho et al. 2005; Jean Piaget, 1885 e Paulo Freire, 1988). Uma AP ajuda a estruturar os processos de aprendizagem, auxiliando os estudantes a construírem seu conhecimento.

Conforme a definição pragmática apresentada em (Reinoso e Tavares, 2015), uma AP é definida pelo conjunto dos componentes: 1. Objetivo de aprendizagem (o que 
VI Congresso Brasileiro de Informática na Educação (CBIE 2017)

Anais do XXVIII Simpósio Brasileiro de Informática na Educação (SBIE 2017)

aprender); 2. Atividades (o que fazer); 3. Método (como desenvolver as atividades); e 4. Recursos digitais (com quais ferramentas).

O framework CAP 1.0, foi a primeira versão da ferramenta digital da pesquisa concebida em concordância com o modelo e conceitos presentes no MOrFEu, um Multi-Organizador Flexível de Espaços virtuais (Menezes et al., 2008; Santos et al., 2010). O modelo CAP permite que o professor crie e edite uma Arquitetura Pedagógica (AP), configurando os recursos que estarão presentes.

A versatilidade do modelo CAP e do Framework CAP 1.0 gerado a partir do mesmo, foi testada por meio da criação de diferentes plataformas CAPs (construtores de arquiteturas pedagógicas) para contextos distintos: aprendizagem de LIBRAS (Língua Brasileira de Sinais) e operações sobre dados multimídia. Com os experimentos, foi verificado que o modelo CAP tem potencial para criação de muitos ambientes didáticos, bastando criar ou ajustar diferentes bibliotecas de recursos para cada contexto.

O restante deste artigo está estruturado da seguinte forma: a seção 2 descreve uma framework para criação e uso de arquiteturas pedagógicas virtuais, sua ideologia e estrutura de projeto; a seção 3 apresenta o CAP-MVLIBRAS (Reinoso et al., 2015), o primeiro Construtor de Arquiteturas Pedagógicas gerado pelo CAP 1.0; a seção 4 apresenta o CAPCOM (Almeida et al., 2016), o segundo Construtor de Arquiteturas Pedagógicas gerado pelo CAP 1.0; a seção 5 apresenta os testes e resultados obtidos; e a seção 6 apresenta as considerações finais e a conclusão do trabalho.

\section{Um Framework para criação e uso de arquiteturas pedagógicas virtuais}

O Framework Construtor de Arquiteturas Pedagógicas (Framework CAP 1.0) possibilita a criação de arquiteturas pedagógicas virtuais, permitindo a um professor, mesmo leigo em informática, gerar um conjunto de atividades e implementar uma AP com recursos digitais que a suportem. O Framework permite ao professor alocar recursos e configurálos, do modo mais adequado, para suportar a AP especificada por ele.

As funções disponíveis em um Construtor de Arquiteturas Pedagógicas (CAP) permitem, ao professor, definir, e aos aprendizes, realizarem atividades individuais ou colaborativas. Esta seção apresenta o modelo MOrFEu (Multi-Organizador Flexível de Espaços Viruais) e conceitos que ajudaram a modelar o CAP, bem como a estrutura e arquitetura de um Framework de desenvolvimento.

\subsection{MorFEu}

O MOrFEu - Multi-Organizador Flexível para Espaços virtuais, surge para garantir um modelo para criação de sistemas que consiga fundamentar a produção de espaços virtuais/digitais de ensino onde seus utilizadores conseguem gerenciar seu conhecimento, pautando quatro aspectos principais: plasticidade, ergonomia, redução da repetição de trabalho e redução da sobrecarga cognitiva (Menezes et al., 2008).

Visando a compreensão de como o MOrFEu modela uma aplicação, devemos conhecer três elementos principais: a Unidade de Produção Intelectual (UPI), o Veículo de Comunicação (VCom) e o Template de VCom.

Segundo Maia Jr. (2011) "O conceito de UPI permite que todo material produzido pelo usuário esteja registrado e versionado de forma independente das publicações". Uma UPI é a unidade-base de toda informação produzida, nela a autoria e 
VI Congresso Brasileiro de Informática na Educação (CBIE 2017)

Anais do XXVIII Simpósio Brasileiro de Informática na Educação (SBIE 2017)

as características do texto são preservadas. Uma UPI pode assumir zero ou muitas associações com VComs.

Segundo Nascimento et al. (2012), uma UPI deve ser enxergada como uma propriedade que garanta a formalização/padronização das atividades dos usuários, permitindo que uma UPI possa ser publicada em diferentes formas de apresentação. Para tanto se faz necessário uma espécie de template (Template de VCom) que consiga padronizar o conteúdo, facilitando a apresentação e utilização da parte visual do sistema.

Um VCom é um canal para troca de comunicações entre pessoas que nele assumem papéis, às vezes simultaneamente, de criadores e consumidores de conteúdo (Pereira et al., 2015). Alguns exemplos que materializam o conceito de VComs são: aplicativos de publicação de mensagem, blogs da internet, redes sociais digitais, revistas, relatório de uma aula de campo, mapas mentais etc.

A composição de várias UPIs é tratada em um Veículo de Comunicação (VCom). Nesta linha, podemos imaginar, a exemplo, um quadro digital de informações escolares. Cada nova informação anexada neste quadro contempla um novo dado acerca do que está ocorrendo com a escola, ou seja, uma UPI criada e inserida por uma pessoa, nesse exemplo, o quadro é um VCom.

$\mathrm{O}$ conceito de template para VCom permite configurar diferentes formas visuais para um mesmo conjunto de UPIs associados a um VCom. Segundo Maia Jr. (2011), "Cada VCom pode estar associado a diferentes templates, permitindo que cada usuário possa definir como deseja visualizá-lo”.

\subsection{Projeto de sistema do Framework CAP 1.0}

A Figura 1 apresenta o modelo de arquitetura do CAP, nele o professor cria uma AP, durante processo de criação, o professor configura os recursos a serem utilizados.

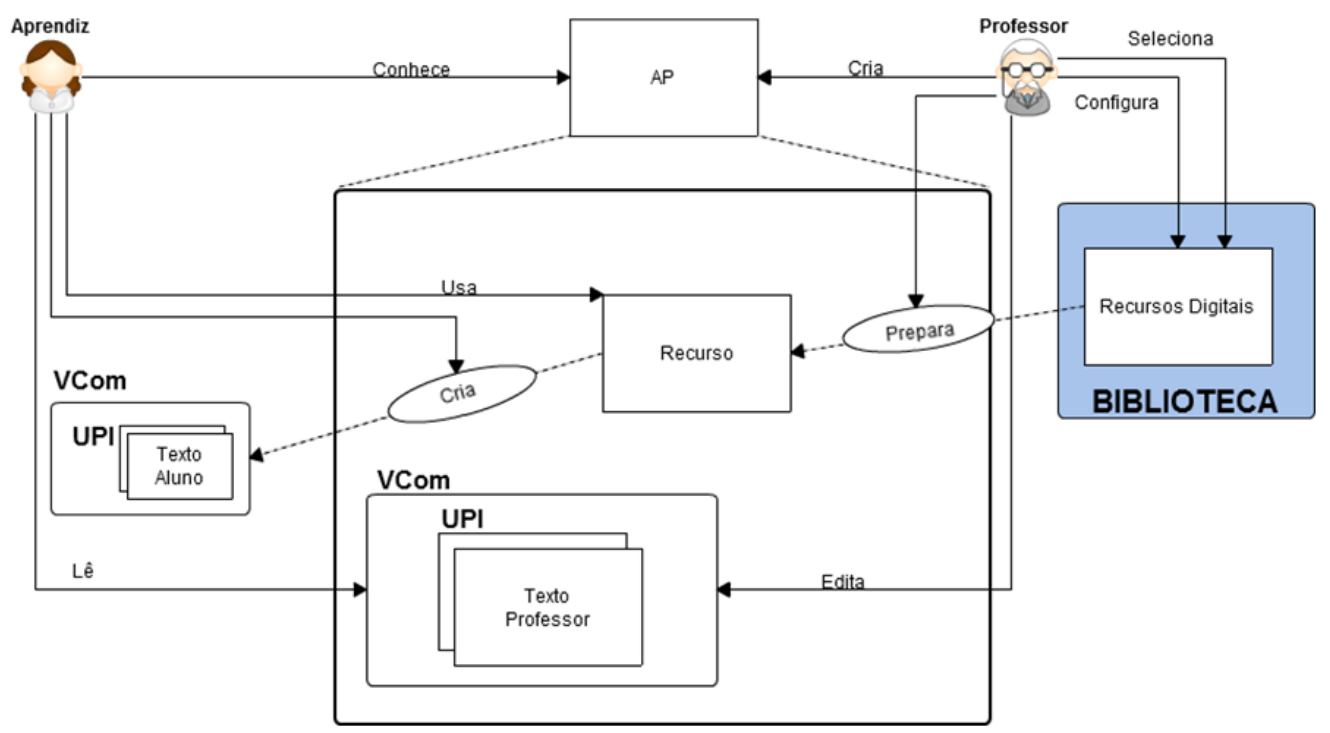

Figura 1. Modelo de arquitetura do CAP.

Cada nova AP constitui-se de UPIs e recursos digitais. As UPIs da AP definem o objetivo pedagógico, as atividades e sua metodologia. Essas UPIs são organizadas em 
VI Congresso Brasileiro de Informática na Educação (CBIE 2017)

Anais do XXVIII Simpósio Brasileiro de Informática na Educação (SBIE 2017)

um VCom (com UPIs e os respectivos recursos digitais), que por fim, estrutura a AP.

Os recursos digitais (armazenados em uma biblioteca) foram implementados na CAP-MVLIBRAS (Reinoso et al., 2015) e na CAPCOM (Almeida et al., 2016) de acordo com a demanda de cada professor envolvido, por serem externos às APs como mostrado na Figura 1, quando um novo recurso é criado/atualizado ele não afeta composições dos alunos e professores, quando o professor seleciona e configura o recurso, essas informações são armazenadas em uma instância criada para a nova AP, ajudando nas etapas de manutenção e atualização das plataformas desenvolvidas, utilizando o modelo CAP, sem prejudicar as APs existentes.

Utilizando o modelo CAP é possível construir uma plataforma que sirva para qualquer AP, independente de área pedagógica. Para atender diferentes contextos é necessário substituir ou complementar a biblioteca de recursos digitais da plataforma, de modo a oferecer os recursos adequados a cada AP criada, uma biblioteca pode servir a uma ou muitas APs distintas.

A metodologia difundida para prospecção de requisitos para o desenvolvimento do Framework CAP 1.0 foi a etnografia (Cervo et al., 2007), que respeita a inteligência e experiência local, logo a partir de entrevistas e conversas com professores e alunos, bem como participação nas atividades de LIBRAS e Português (CAP-MVLIBRAS) e em atividades de campo (CAPCOM).

O framework foi desenvolvido a partir do paradigma de desenvolvimento orientado a objetos, nesse tipo de implementação os objetos que compõem o sistema interagem entre si. O ciclo de vida incremental foi adotado para o projeto (Pressman, 2011).

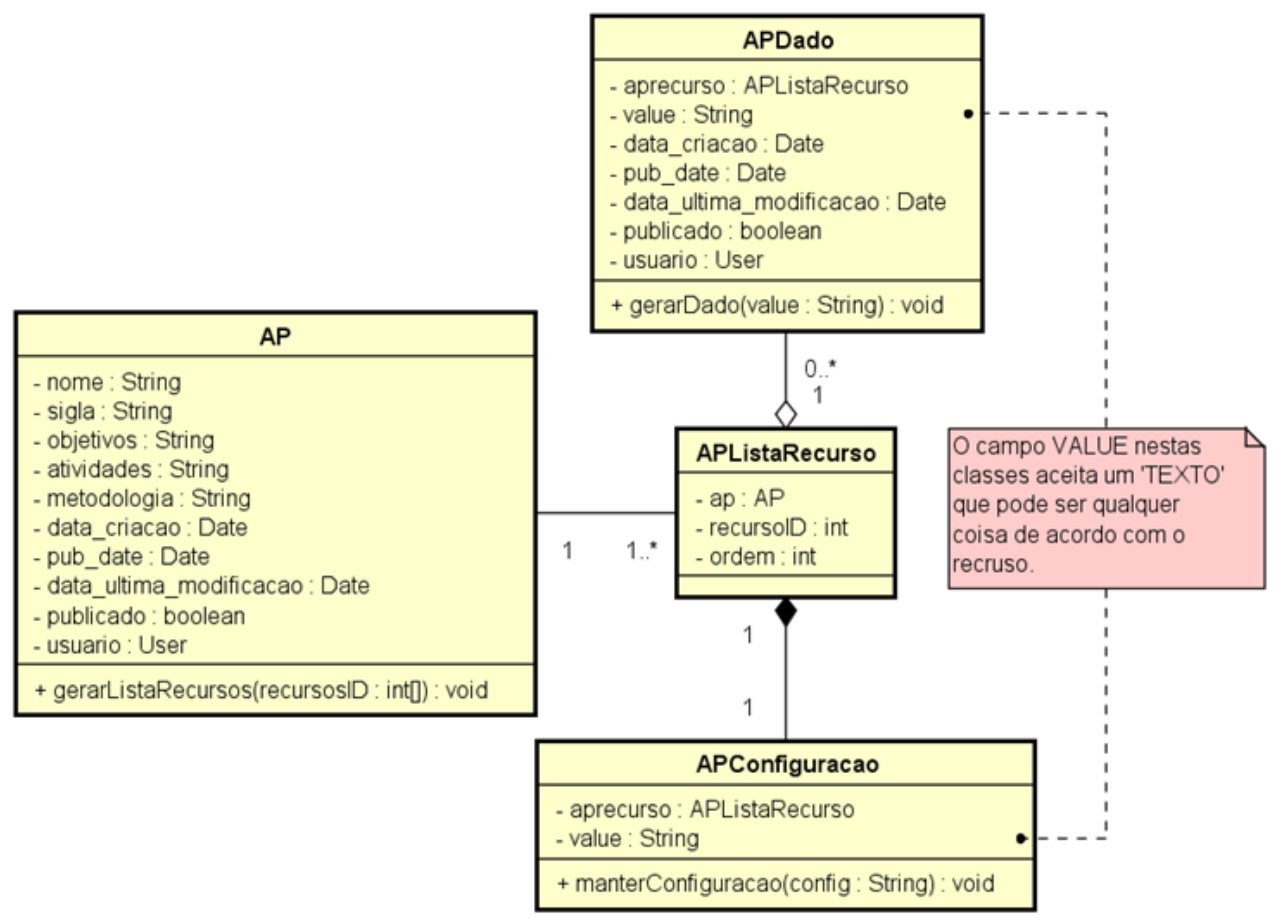

Figura 2. Diagrama de classes da Framework CAP.

O funcionamento do modelo apresentado (Figura 1) fundamentou a criação do Framework CAP 1.0, o modelo de classes com os atributos e relações necessárias ao seu 
VI Congresso Brasileiro de Informática na Educação (CBIE 2017)

Anais do XXVIII Simpósio Brasileiro de Informática na Educação (SBIE 2017)

funcionamento, apresentado na Figura 2 é descrito a seguir:

1. Classe AP: cada objeto da classe AP possui os atributos: nome, sigla (nome curto), o criador da AP, os objetivos de aprendizagem, as atividades pedagógicas, a metodologia e os recursos digitais necessários.

2. Classe APListaRecurso: descreve os recursos digitais, selecionados pelo criador da AP, a serem usados no desenvolvimento das atividades. Para cada recurso digital alocado, é instanciado um objeto da classe APConfiguracao.

3. Classe APConfiguracao: define as características dos objetos de configuração dos recursos digitais. Assim, cada recurso digital pode ser configurado de diferentes modos.

4. Classe APDado: descreve as UPIs criadas pelos aprendizes com o uso dos recursos digitais.

As classes de objetos APConfiguracao e APDado têm um campo 'value' do tipo TEXTO, que pode ser qualquer valor. Assim, seguindo as diretrizes de um CAP, cada recurso digital acoplado pode receber valores diferentes para seus parâmetros. Cada recurso trata esses valores para se comportar do modo configurado.

\section{CAP-MVLIBRAS}

Para o desenvolvimento da plataforma CAP-MVLIBRAS, utilizando o Framework CAP 1.0, seu modelo e ideologia, contamos com um estudo Etnográfico, partindo de análises de documentos institucionais e atividades da disciplina 'Introdução a LIBRAS', área de aprendizagem utilizada para teste do conceito, acompanhado de um professor (intérprete surdo) e seus alunos, de um curso técnico de 'Tradução e interpretação LIBRAS'.

Neste contexto foram realizadas um conjunto de atividades de modelagem do sistema, baseado no levantamento de APs até a fundamentação do projeto para criação do protótipo funcional.

As APs foram licitadas pelo professor, estas possibilitaram a implementação de recursos digitais diversificados e complementares as necessidades do mesmo, em contrapartida a seus objetivos e atividades pedagógicas. Definiu-se então as seguintes APs que a plataforma atende:

- APAVeL - Arquitetura Pedagógica para Aprendizagem de Verbos em LIBRAS: o objetivo pedagógico dela é permitir a aprendizagem sobre a execução da sinalização gestual animada de verbos da LIBRAS. A atividade associada é: cada usuário dessa AP deve fazer a gesticulação animada de verbos de uma lista de verbos. A metodologia é: gravar a gesticulação animada de cada verbo. Os recursos digitais alocados e disponíveis na AP permitem: gravar em vídeo as gesticulações dos alunos.

- $\mathrm{APAGeV}$ - Arquitetura pedagógica para aprendizagem da gesticulação animada de verbos em LIBRAS: o objetivo pedagógico dessa AP é a interpretação de verbos sinalizados em LIBRAS. A atividade prevista aqui é escolher e visualizar verbos sendo estes sinalizados e animados em LIBRAS. A metodologia: pesquisar e buscar a datilologia e gesticulação de verbos do Português em LIBRAS, contidos dentro de bibliotecas e dicionários. Os recursos digitais disponíveis na AP ajudam na busca por sinais e visualização de palavras 
VI Congresso Brasileiro de Informática na Educação (CBIE 2017)

Anais do XXVIII Simpósio Brasileiro de Informática na Educação (SBIE 2017)

sinalizadas em LIBRAS.

- APTGeF - Arquitetura pedagógica para tradução e gesticulação de frases: o objetivo pedagógico da AP é a tradução de sentenças em Português para LIBRAS gestual. A atividade: os alunos fazem a tradução de uma frase em Português para LIBRAS gesticulada, traduzindo e sinalizando simultaneamente. A metodologia: uma frase escrita elaborada pelo professor é passada para os aprendizes realizarem sua tradução e gesticulação em LIBRAS. A AP aloca recursos digitais: que permitam gravar a sinalização em LIBRAS das sentenças em Português.

- APIL - Arquitetura pedagógica para interpretação visual em LIBRAS: o objetivo pedagógico é permitir a interpretação da LIBRAS gesticulada e permitir sua tradução para o Português escrito. A atividade: os alunos devem interpretar gestos de um vídeo do professor em LIBRAS e escrever a tradução da interpretação em Português. A metodologia: os aprendizes visualizam um texto sinalizado em LIBRAS, o interpretaram e transcrevem a tradução do texto para Português. Os recursos digitais para esta AP: campos de entrada de texto com a resposta dos vídeos do professor em Português.

Após a especificação das APs acima, foi possível projetar e implementar os recursos digitais previstos em cada uma delas. Esses recursos digitais foram projetados de modo a ficarem flexíveis e permitirem a configuração de vários parâmetros modificadores do comportamento dos recursos. O conjunto desses recursos constituiu uma biblioteca para consumo da Framework CAP 1.0, que passa a integrar a plataforma CAP.

A Framework CAP 1.0 suporta o uso de cada AP permitindo aos alunos o acesso aos recursos digitais alocados na AP. Os recursos digitais da biblioteca podem ser usados e configurados em diferentes APs.

\section{4. САРСОМ}

A CAPCOM (Construtor de Arquiteturas Pedagógicas Colaborativas para Operações Cognitivas sobre Dados Multimídia), foi projetado para permitir a execução de operações cognitivas sobre dados multimídia, com o objetivo de auxiliar os alunos no processo de consolidação de aprendizado adquirido em uma aula de campo.

Utilizando a CAPCOM, uma ou várias APs podem ser criadas, o educador configura os recursos que serão utilizados na AP. Os recursos são bibliotecas para processar diferentes tipos de mídias (UPIs), combinado com suporte à configuração que permite modificar o comportamento do recurso digital utilizado (Almeida, Tavares e Reinoso, 2016).

Para avaliar o potencial da plataforma CAPCOM no contexto pós-aula de campo, foram propostas seis arquiteturas pedagógicas, que permitem os alunos executaram operações cognitivas sobre dados multimídia, as AP propostas são:

- Arquitetura Pedagógica Cadeia Alimentar. Objetivo pedagógico: Aprender sobre os níveis tróficos de uma cadeia alimentar, dado um conjunto de seres vivos de um ecossistema. Atividades: Associar mídias produzidas em uma aula de campo, usando mapas mentais como interface para visualizar as associações. Método: O professor seleciona um conjunto de mídias que precisa ser explorado 
pelos alunos. O professor forma grupos de três alunos e solicita que os alunos colaborem para associar todas as mídias produzidas em uma aula de campo. Recurso digital: São usados recursos para visualizar mapas mentais, classificação de dados multimídia, suporte a colaboração, chat e grupo.

- Arquitetura Pedagógica Relato de Grupo. Objetivo pedagógico: Aprender a criar um relato colaborativo com os dados multimídia da aula em campo. Atividades: Combinar logicamente os dados multimídia e produzir um relatório das atividades desenvolvidas durante a aula de campo. Método: O professor forma grupos de cinco alunos e desafia o grupo a produzir um relatório das atividades conduzidas durante a aula de campo, usando os dados multimídia produzidas durante a atividade. Recurso digital: São utilizados recursos para edição de dados multimídia, suporte à colaboração, chat, grupo e negociação para uso de dados multimídia.

- Arquitetura Pedagógica Fórum de Vídeos. Objetivo pedagógico: Aprender a registrar e processar vídeos. Atividades: Combinar os vídeos de forma sequencial e registrar os vídeos. Método: O professor define que a atividade será em grupo; define que só UPIs do tipo vídeo podem ser utilizadas; forma grupos de cinco alunos e desafia os alunos a se ajudarem para registrar seus vídeos de forma sequencial. Recurso digital: São usados recursos para edição de dados multimídia no formato de relato, suporte à colaboração, chat, grupo, negociação para uso de dados multimídia e registro de novos dados multimídia.

- Arquitetura Pedagógica Associando Conceito. Objetivo pedagógico: Aprender a classificar dados multimídia, de acordo com os conceitos propostos pelo professor. Atividades: Associar textos produzidos em uma aula de campo a um conceito e visualizar as associações no formato de mapas mentais. Método: $\mathrm{O}$ professor cria um conceito que precisa ser explorado pelos alunos, forma grupos de três alunos e solicita que os alunos colaborem para associar todas as mídias produzidas na aula de campo, que tem relação com o conceito. Recurso digital: São utilizados recursos para visualizar mapas mentais, classificação de dados multimídia, suporte a grupos de colaboração, chat, grupo e edição de dados multimídia.

- Arquitetura Pedagógica Guia Digital. Objetivo pedagógico: Aprender a descrever a experiência de aprendizado vivenciada durante uma aula de campo. Atividades: Combinar logicamente os dados multimídia e produzir uma apresentação das atividades desenvolvidas durante a aula de campo. Método: $\mathrm{O}$ professor cria um grupo de três alunos e desafia o grupo a produzir uma apresentação das atividades conduzidas durante a aula de campo. Recurso digital: São utilizados recursos para edição de dados multimídia, suporte a colaboração, chat, grupo e negociação para uso de dados multimídia.

- Arquitetura Pedagógica Fórum de Fotografia Esférica. Objetivo pedagógico: Aprender a registrar e processar fotos esféricas. Atividades: Combinar e registrar fotos esféricas. Método: O professor define que a atividade será em grupo; define que só UPIs do tipo fotos esféricas podem ser utilizadas; forma grupos de cinco alunos e desafia os alunos a se ajudarem para registrar suas fotos esféricas de forma sequencial. Recurso digital: São utilizados recursos para edição de dados multimídia, suporte a colaboração, negociação para uso dados multimídia, 
VI Congresso Brasileiro de Informática na Educação (CBIE 2017)

Anais do XXVIII Simpósio Brasileiro de Informática na Educação (SBIE 2017)

chat, grupo e registro de novos dados multimídia.

\section{Testes e resultados obtidos}

A validação do Framework CAP foi feita por meio de estudos de caso. No experimento relacionado com o CAP-MVLIBRAS foram testados os aspectos funcionais e sua usabilidade, em uma turma com um professor interprete (surdo) e 36 estudantes. O segundo experimento envolveu o CAPCOM, realizado com um grupo de sete pessoas, sendo dois professores e cinco estudantes. A análise realizada neste último experimento foi feita de acordo com uma metodologia de pesquisa quantitativa.

Nos testes realizados com o CAP-MVLIBRAS, $13.88 \%$ dos estudantes relataram existir diferenças entre a prática de suas atividades habituais e a realização delas na plataforma CAP, enquanto 5.66\% relataram alguma dificuldade para realizar atividades no ambiente virtual. A avaliação média para o fator usabilidade do sistema foi de $88 \%$.

O CAP-MVLIBRAS passou também por um teste funcional, também chamado de teste baseado em especificações ou teste caixa-preta (Pezzè e Young, 2008). Os alunos foram submetidos a utilizar a plataforma no intuito de encontrar melhorias entre os recursos da plataforma e das APs dispostas pelo professor, que também avalia a ferramenta de seu ponto de vista. Das disposições gerais dadas pelos alunos e seu professor, após avaliação dos dados gerados por eles nos testes, temos:

- O sistema funciona inteiramente com os recursos digitais;

- A turma sente falta de recursos de inclusão, como sistemas de tradução com avatar;

- Os alunos acreditam que ter recursos assistivos, ajudaria e até mesmo incentivaria a inclusão da comunidade de surdos na plataforma. (Ressalta-se que os alunos da turma participante desta pesquisa são todos ouvintes).

O teste funcional revelou que os utilizadores da CAP-MVLIBRAS indicam a necessidade de aperfeiçoar os recursos digitais, mas a plataforma em si funcionou inteiramente. Entretanto, é visível a dependência das APs aos recursos especialmente desenvolvidos para atendê-las. Esse fato justifica a possibilidade de variação (diferentes configurações) de um mesmo recurso, através de ações de configuração pelo professor, como apresentado na Figura 1.

Nos testes realizados a partir do CAPCOM foram aplicados, a uma turma de 12 alunos do ensino médio e ao professor da turma, um questionário sobre o conteúdo das atividades pedagógicas desenvolvidas em campo. O formulário era composto por 29 questões objetivas, 15 verdadeiras e 14 falsas, obtendo $76 \%$ das respostas fornecidas pelos estudantes como corretas.

As validações ocorreram com um grupo reduzido de pessoas, os números traduzem um indicativo de que o uso da Framework CAP 1.0, seguindo o modelo CAP, tem o potencial de favorecer a fixação de conhecimento nos contextos trabalhados e de facilitar a criação de atividades com o uso de recursos digitais por professores leigos em informática.

\section{Considerações finais}


Os conceitos de Arquiteturas Pedagógicas, usados na concepção do modelo CAP, ajudaram a entender como planejar e estruturar as atividades pedagógicas, de modo que seguindo uma metodologia e usando os recursos digitais disponíveis, os alunos possam alcançar um objetivo de aprendizagem pretendido pelo professor. Os conceitos do projeto MOrFEU ajudaram a conceber uma arquitetura para a CAP que permita a alocação de recursos digitais, configuráveis pelo professor e alocados a cada AP, atendendo aos requisitos dessa AP. Além disso, os conceitos de UPI, VCom e template permitiram o desenvolvimento de uma plataforma para construção de arquiteturas pedagógicas que incorpora de modo inovador os conceitos de flexibilidade, reuso e persistência dos dados (UPIs).

Um CAP funcional é uma plataforma para a construção e uso de arquiteturas pedagógicas para a aprendizagem. Os recursos inseridos em uma plataforma CAP reduziram de forma significativa o tempo e a dificuldade de se criar arquiteturas pedagógicas para aprendizagem de LIBRAS e atividades de campo.

Os conceitos de Arquiteturas Pedagógicas, usados na concepção do modelo $\mathrm{CAP}$, ajudaram a entender como planejar e estruturar atividades pedagógicas, de modo que, seguindo uma metodologia e usando os recursos digitais disponíveis, os alunos possam alcançar o objetivo de aprendizagem pretendido pelo professor.

A versatilidade do modelo CAP e seu Framework CAP 1.0 foram testados na modelagem de duas plataformas com focos distintos, ambos permitindo criar arquiteturas pedagógicas diversas, de acordo com o objetivo do educador. Com a implementação em contextos distintos, o modelo CAP demonstra que tem o potencial de atender as necessidades de diversas APs, bastando criar ou ajustar as bibliotecas para cada contexto.

A Framework CAP 1.0 e as suas instâncias, CAP-MVLIBRAS e CAPCOM desenvolvidas neste trabalho são de uso livre, sendo disponibilizada para editoração de APs e desenvolvimento.

\section{Referências}

Almeida, R., Tavares, O. L., Reinoso, L. F. (2016) "CAPCOM - Construtor de Arquiteturas Pedagógicas para Dinâmicas Colaborativas com Multimídia. In: SBSC 13 $3^{\circ}$ Simpósio Brasileiro de Sistemas Colaborativos”. In: XXXVI Congresso da Sociedade Brasileira de Computação. Porto Alegre: SBC, pages 1423-1436.

Carvalho, M. J. S., Nevado R. A. and Menezes, C. S. (2005) “Arquiteturas Pedagógicas para Educação a Distância: Concepções e Suporte Telemático. In XVI Simpósio Brasileiro de Informática na Educação”. Páginas 351-360, 2005.

Cervo, A. L., Bervian, P. A. and Silva, R. (2007) "Metodologia Científica". 6a ed. São Paulo: Pearson Prentice Hall.

Freire, P.(1998) Pedagogia da Autonomia: saberes necessários à prática educativa. Rio de Janeiro: Paz e Terra.

Maia Jr., R. G. (2011). "Uma arquitetura de software para o MOrFEu: apoiando a realização de arquiteturas pedagógicas em espaços virtuais colaborativos”. Master's thesis, Programa de pós-graduação em Informática da UFES, Vitória-ES.

Menezes, C. S., Nevado, R. A., Junior, A. N. C. and Santos, L. N. (2008) “MOrFEu - 
VI Congresso Brasileiro de Informática na Educação (CBIE 2017)

Anais do XXVIII Simpósio Brasileiro de Informática na Educação (SBIE 2017)

Multi-Organizador Flexível de Espaços Virtuais para Apoiar a Inovação Pedagógica em EAD”. In XIX Simpósio Brasileiro de Informática na Educação. Páginas 451460.

Nascimento, C. V., Menezes. C. S. and Tavares, O. L. (2012) "Uma arquitetura de acessibilidade para ambientes virtuais". In: Memorias del XVII Congresso Internacional de Informática Educativa, TISE, Santiago-Chile, pages 353-358.

Pereira, B., Almeida, R., Tavares, O. L. and Menezes, C. S. (2015) “AM: Uma Plataforma Digital para Criação e Uso de Veículos de Comunicação Locativos para Atividades Pedagógicas." In Anais do Simpósio Brasileiro de Informática na Educação. Vol. 26. No. 1.

Pezzè, M. and Young, M. (2008) "Teste e análise de software: processos, princípios e técnicas”. Porto Alegre: Bookman, 512p.

Piaget, J. (1985). O possível e o necessário - evolução dos possíveis na criança. Porto Alegre, Artes Médicas.

Pressman, R. S. (2011) “Engenharia de Software: Uma abordagem Profissional”. ed. 7, Porto Alegre: AMGH, p. 780.

Reinoso L. F. and Tavares, O. L. (2015) "MVLIBRAS: ambiente digital para comunidades de aprendizagem com recursos inclusivos para surdos". In: Anais do XXVI Simpósio Brasileiro de Informática na Educação. Págs 772-881. MaceióAlagoas.

Santos, L. N., Jr, A. N. C. and Menezes, C. S. (2010) "MOrFEu: criando ambientes virtuais flexíveis na WEB para mediar a colaboração". In Congresso Ibero-americano de Informática Educativa. Páginas 114-121. 